\title{
Measles Virus is Associated with Hodgkin Lymphoma and Additional Tumors - a Never Ending Story
}

\author{
Daniel Benharroch* \\ Department of Pathology, Soroka University Medical Center, Israel
}

*Corresponding author: Daniel Benharroch, Retired, Formerly, Head of Hematopathology Unit, Department of Pathology, Soroka University Medical Center and Faculty of Health Sciences, Ben-Gurion University of the Negev, Beer-Sheva, Israel

To Cite This Article: Daniel Benharroch, Measles Virus is Associated with Hodgkin Lymphoma and Additional Tumors - a Never Ending Story. Am J Biomed Sci \& Res. 2021 - 12(1). AJBSR.MS.ID.001712. DOI: 10.34297/AJBSR.2021.12.001712.

Received: 紫 February 02, 2021; Published: 筒 February 19, 2021

\section{Introduction}

\section{"Ancient history" microbes and cancer}

About $15 \%$ of human cancers -thought to arise by mechanisms that involve viruses, bacteria or parasites.

Evidence for their involvement - partly from the detection of the agents in biopsies.......and partly from animal and epidemiological studies. The biologic agent (virus) is usually responsible for only a limited number of steps in the initiation/progression of cancer. In many cases the precise role of cancer-associated virus is hard to decipher due to the long delay from the initial viral infection! The number of individuals infected by the virus is much larger than that with virus-associated cancers. Therefore, viruses must act in conjunction with other factors

\section{Chronic inflammation and cancer}

In some cases of unresolved chronic inflammation, the immune response becomes maladaptive, promoting tumorigenesis. A regenerative process supported by numerous bioactive mediators, promote cell survival, tissue remodeling and angiogenesis. The mediators also cause genomic stress and mutations.

\section{Hodgkin lymphoma}

Hodgkin lymphoma is a cancer of lymph nodes and of the immune system. Infrequent, but one of the most frequent cancers of young adults (15-34). As a rule, very few cancer cells in tumor mass. Very good response to treatment ( $80 \%$ and more are cured).

\section{Epidemiologic correlation of EBV in classic Hodgkin lymphoma}

The association of EBV in cHL varies from $17 \%$ in some industrialized countries to $100 \%$ in a few developing countries.
EBV expression varies with gender:

Male patients are more likely to be EBV positive than are female patients (figure 1).

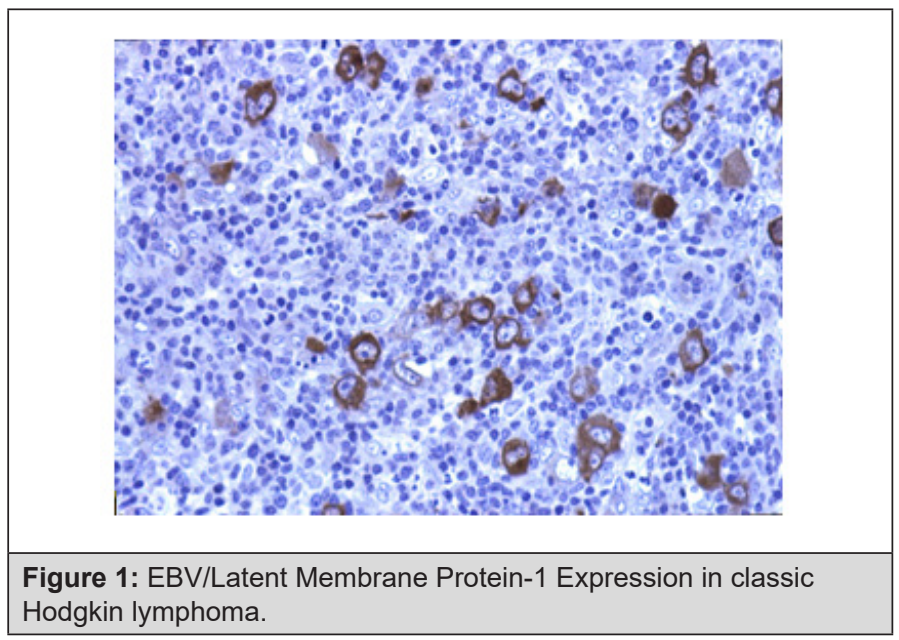

EBV expression varies with histologic subtype:

a) Mixed cellularity (32-96\%)

b) Nodular sclerosis (10-50\%)

\section{Pathogenesis of EBV-related cHL}

EBV-associated NF- $\kappa B$ activation may lead to cell proliferation, inflammation, and apoptosis arrest EBV/LMP1 has a Bcl-2-like effect and causes apoptosis resistance.

a) Children mostly EBV-positive males with MCHL (figure 2).

b) Young adults (both sexes) mostly EBV-negative NSHL

c) Young adults with previous mononucleosis, EBV positive 
d) Older adults mainly EBV positive males with MCHL

\section{Thus, EBV may account for only part of cHL cases}

Young-adult cHL patients, mainly women, who are those most expected to be related with late exposure to a common infection (according to hallmark epidemiologic studies) have the lowest link to EBV. None of the additional viruses, considered so far as possible candidates, have been found in cHL tissues.

In favor of a measles virus-association with classic Hodgkin lymphoma

a) Measles virus is lymphotropic

b) It also shows tropism for the oropharyngeal epithelium

c) Measles infection is regularly followed by transient immune deficiency d) Multinucleated giant cells are seen in measles and in cHL

e) Since 1963, following anti-MV vaccination, measles infection occurs more often after age 5

We have proposed the measles virus as a possible biologic agent related to classic Hodgkin lymphoma, at least in young adult patients.

\section{Patients and Methods}

a) 154 biopsies from untreated cHL patients

b) IHC study in paraffin sections - avidin-biotin peroxidase complex method

c) Reverse Transcriptase-PCR on total RNA from frozen and paraffin-embedded tissues

d) Products of nested PCR were blotted in situ hybridization - according to Ogata et al. (figure 3-5).
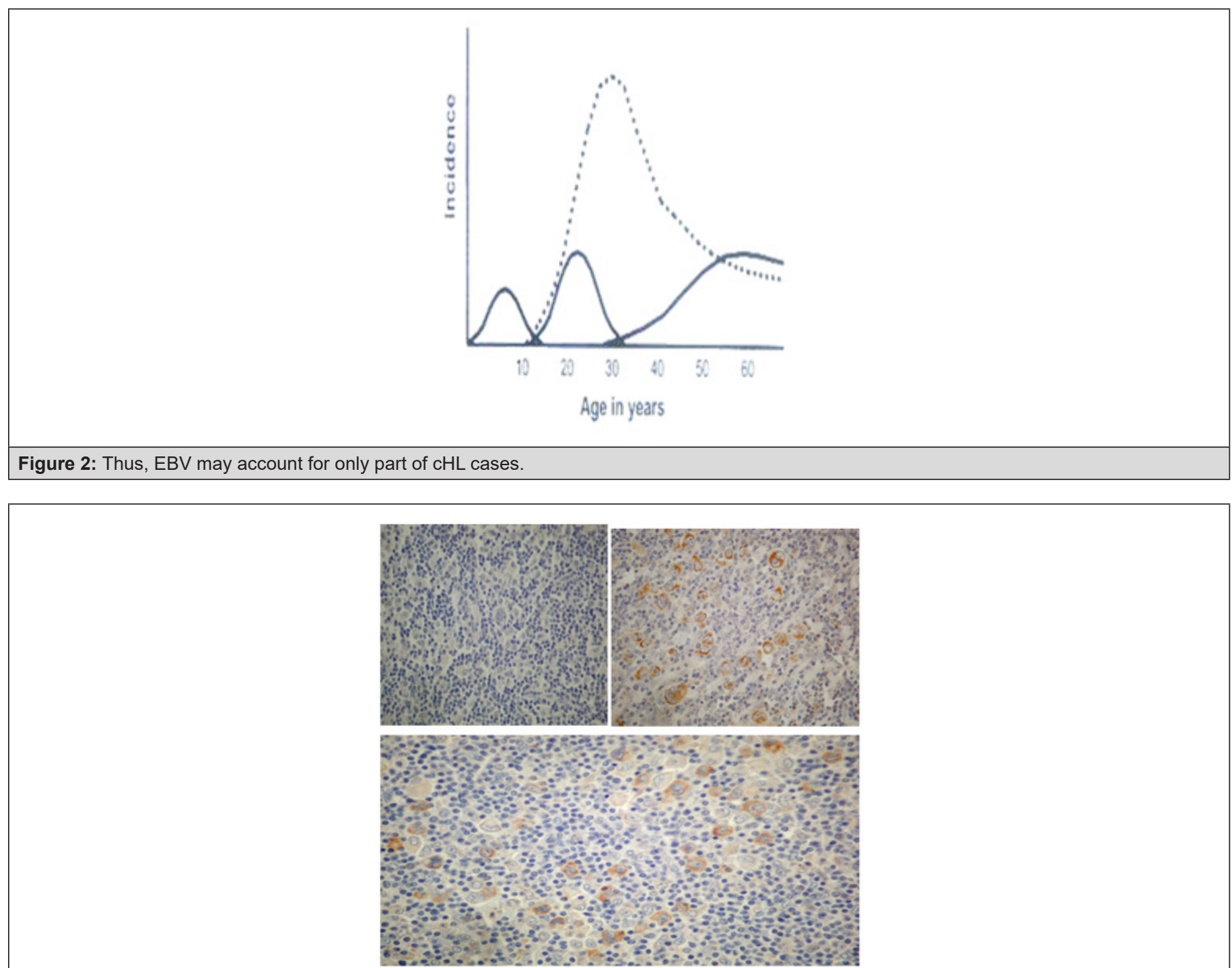

Figure 3(a): Immunohistochemistry. 


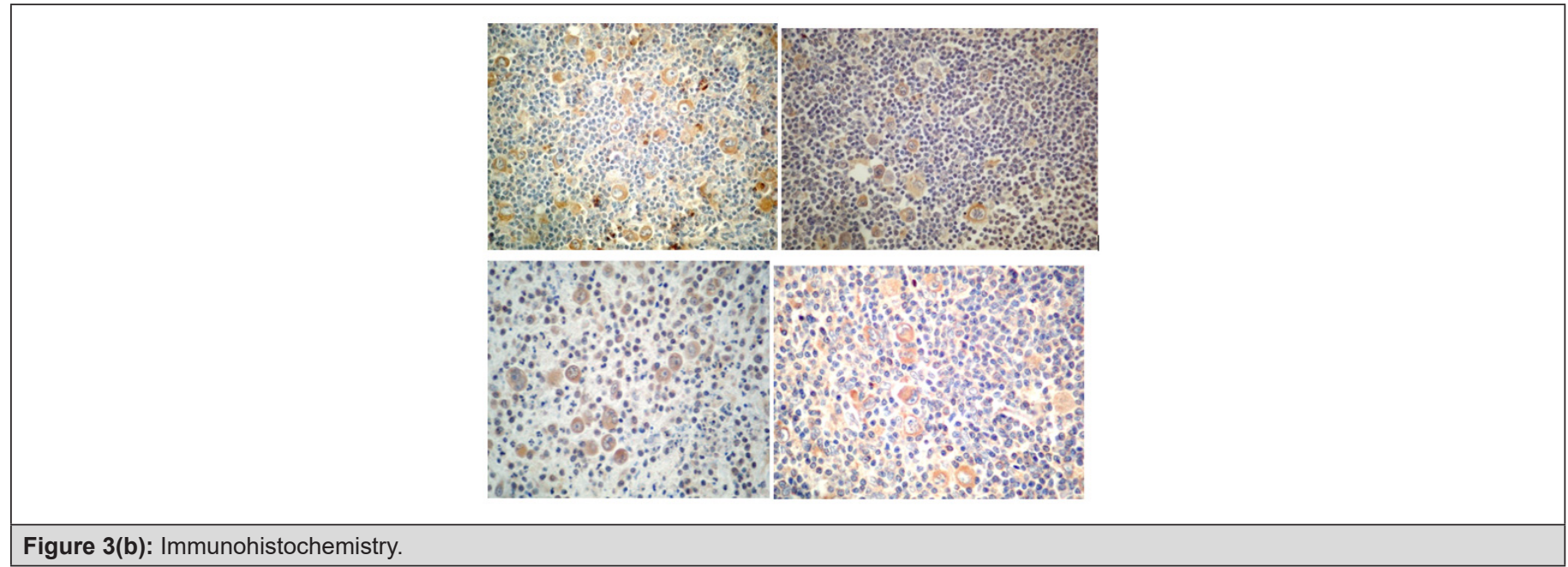

\section{HL}
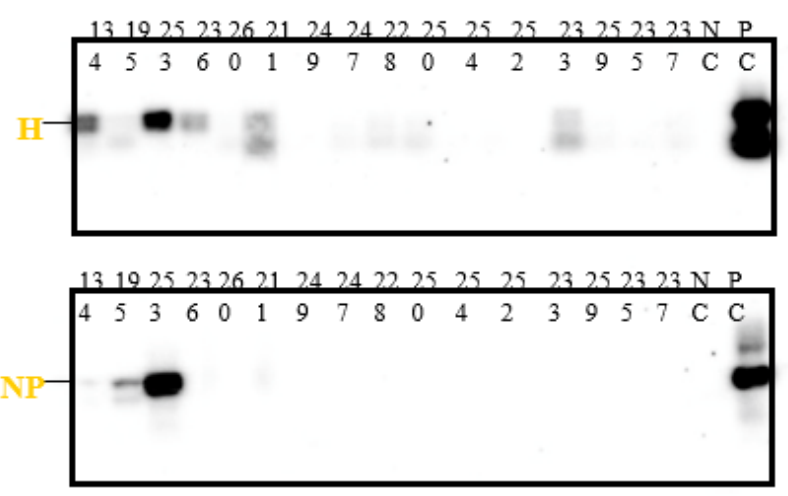

NHL

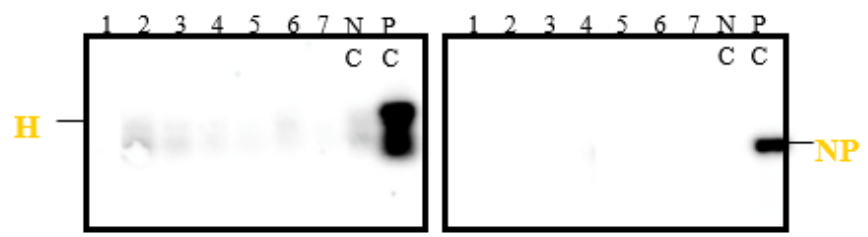

Figure 4: Southern Blot.

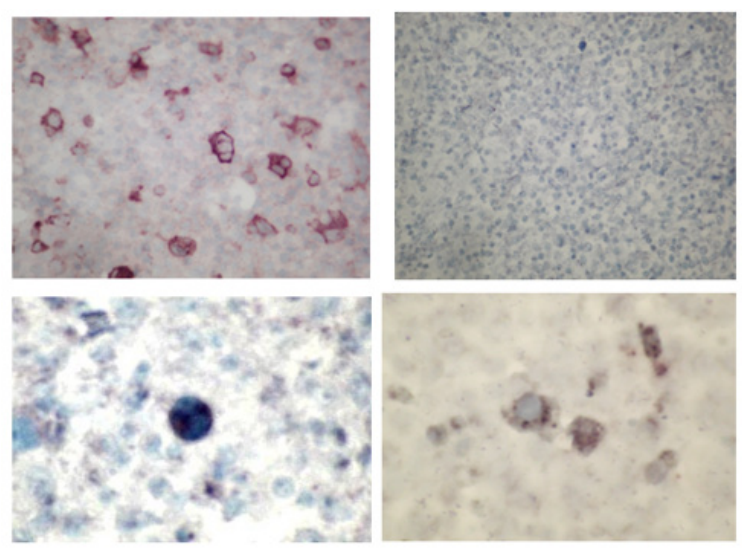

Figure 5: 82 (54\%) were positive for two MV antigens or more HA-MV RNA + (4/15); NP-MV RNA + (2/16) - by Southern blot HA-MV RNA + (2/7); NP-MV RNA + (8/21) - by in situ hybridization. 


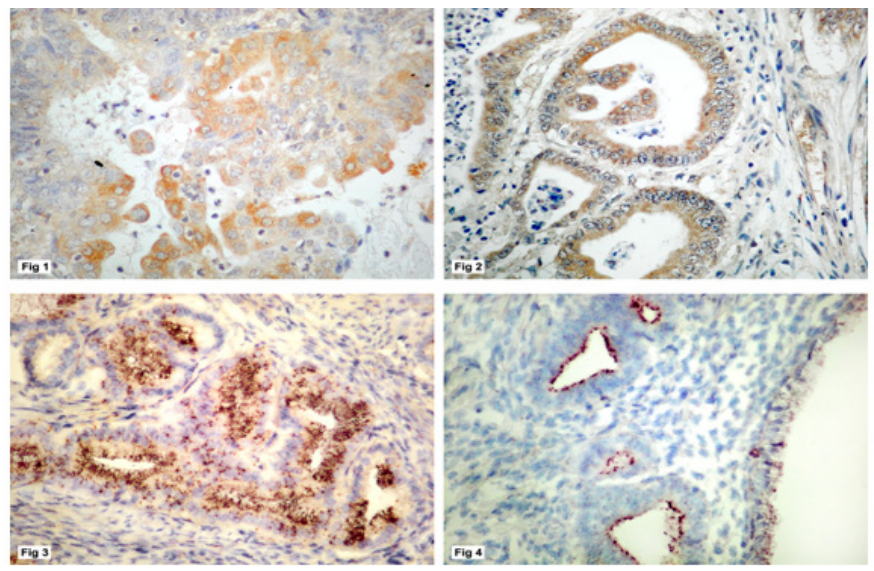

Figure 6: Endometrial Carcinoma and Measles virus.

\section{Results}

a) $82(54 \%)$ were positive for two MV antigens or more.

b) HA-MV RNA + (4/15); NP-MV RNA + (2/16) - by Southern blot.

c) HA-MV RNA + (2/7); NP-MV RNA + (8/21) - by in situ hybridization (table 1-3).

Table 1: Hodgkin lymphoma - Association with MV.

\begin{tabular}{|c|c|c|c|c|}
\hline \multirow{2}{*}{\multicolumn{2}{|c|}{ n (\%) }} & MV + & MV - & p value \\
\hline & & n (\%) & \multirow{2}{*}{$69(45.7)$} & \\
\hline \multicolumn{2}{|c|}{ Total } & $82(54.3)$ & & \\
\hline \multirow{2}{*}{ Gender } & $\mathrm{F}$ & $40(64.5)$ & $28(35.5)$ & \multirow{2}{*}{0.036} \\
\hline & $\mathrm{M}$ & $42(47.2)$ & $47(52.8)$ & \\
\hline \multirow{2}{*}{ Subtype } & NS & $58(68.2)$ & $27(31.8)$ & \multirow{2}{*}{0.0013} \\
\hline & MC & $21(40.4)$ & $31(59.6)$ & \\
\hline \multirow{2}{*}{ CD15 } & pos & $77(59.7)$ & $52(40.3)$ & \multirow{2}{*}{0.015} \\
\hline & neg & $4(26.7)$ & $11(73.3)$ & \\
\hline \multirow{2}{*}{ p53 } & pos & $72(61.5)$ & $45(38.5)$ & \multirow{2}{*}{0.017} \\
\hline & neg & $5(31.3)$ & $11(68.8)$ & \\
\hline
\end{tabular}

Table 2: Hodgkin lymphoma - Association with Measles and Epstein-Barr viruses.

\begin{tabular}{|c|c|c|c|c|}
\hline & & MV + & MV - & $p$ value \\
\hline & & n (\%) & n (\%) & \\
\hline Total & & $82(54.3)$ & $69(45.7)$ & \\
\hline \multirow{2}{*}{ Gender } & $\mathrm{F}$ & $40(64.5)$ & $28(35.5)$ & \multirow{2}{*}{0.036} \\
\hline & M & $42(47.2)$ & $47(52.8)$ & \\
\hline \multirow{2}{*}{ Subtype } & NS & $58(68.2)$ & $27(31.8)$ & \multirow{2}{*}{0.0013} \\
\hline & $\mathrm{MC}$ & $21(40.4)$ & $31(59.6)$ & \\
\hline \multirow{2}{*}{ CD15 } & pos & $77(59.7)$ & $52(40.3)$ & \multirow{2}{*}{0.015} \\
\hline & neg & $4(26.7)$ & $11(73.3)$ & \\
\hline \multirow{2}{*}{ p53 } & pos & $72(61.5)$ & $45(38.5)$ & \multirow{2}{*}{0.017} \\
\hline & neg & $5(31.3)$ & $11(68.8)$ & \\
\hline
\end{tabular}

Table 3: Multivariable logistic regression model of association of prognostic factors with dying of $\mathrm{HL}$ in 89 patients.

\begin{tabular}{|c|c|c|c|}
\hline Factor & Odds ratio & $\mathbf{9 5 \%}$ CI & p value \\
\hline gender & 1.32 & $.34-5.18$ & 0.69 \\
\hline stage & 13.67 & $2.3-80.5$ & 0.004 \\
\hline
\end{tabular}




\begin{tabular}{|c|c|c|c|}
\hline Bcl-2 & 0.34 & $.08-1.44$ & 0.34 \\
\hline MV+; EBV- & 10.05 & $.98-103.47$ & 0.05 \\
\hline MV- ;EBV+ & 1.53 & $.07-35.44$ & 0.79 \\
\hline MV-;EBV- & 15.2 & $1.05-220.3$ & 0.04 \\
\hline
\end{tabular}

Table 4: New findings": melanoma and measles virus.

\begin{tabular}{|c|c|c|c|}
\hline \multirow{2}{*}{ Antigen } & Level & Melanoma & Benign nevi \\
\hline \multirow{2}{*}{ NP-MV } & pos & 46 & 23 \\
\cline { 2 - 4 } & neg & 10 & 1 \\
\hline \multirow{2}{*}{ P-protein } & pos & 19 & 10 \\
\cline { 2 - 4 } & neg & 36 & 12 \\
\hline \multirow{2}{*}{ HA-MV } & pos & 19 & 7 \\
\cline { 2 - 4 } & neg & 3 & 1 \\
\hline \multirow{2}{*}{ PirH-2 } & pos & 44 & 19 \\
\hline
\end{tabular}

\section{Comments}

We have shown an association between MV and HL (though causality was not proven!) .......supported by epidemiologic data on brain and spinal cord tumors, as well as $\mathrm{cHL}$, following exposure to MV around the time of birth. Sporadic cHL regression is reported after measles or MV vaccination. Childhood infections (including measles!) protect from cHL. Preliminary clinical correlations show more female patients, more NS-cHL and more patients with early stages among MV positive expression in cHL patients.

By contrast, positive MV-expression in cHL seem to be associated with a poor prognosis. It is not excluded that the advantage due to the above-mentioned parameters is nullified by the expression of MV

a) Isolated MV expression and absence of both EBV and MV expression are independent poor prognostic factors.

b) Positive EBV expression is related with a longer survival.

c) The longest survival is related with the positive expression of both viruses.

\section{"Old History" The german connection - Maggio E, Küppers R}

Maggio et al selected 18/44 of their own cHL fresh frozen tissues for their high-quality RNA and very good HRS cell morphology. They used UV-laser beam single cell microdissection. About 100 cells were pooled for each experiment, RNA was extracted, and RTPCR performed with primers from three MV genes.

\section{Results}

The cHL-derived cell lines - L428, L1236, KMH2, HDLM2 were negative for the $3 \mathrm{MV}$ transcripts. The 18 German cases did not show evidence of any of the three MV viral transcripts. GAPDH housekeeper (control!) gene showed strong evidence of RNA.

\section{The Israeli connection}

We sent 22 of our fresh-frozen cHL tissues to Germany, chosen for their positive IHC staining with MV antigens

But only 7 of these were selected for high quality RNA and excellent HRS cell morphology in Essen. These were all found by Maggio et al, to be negative for the various MV transcripts studied. Five of twelve German cases were found by us to show MV antigens expression in HRS cells. A similar discrepancy in favor of IHC obtained also in our patients.

\section{Comments}

In the German study few but highly selective cases were studied using different, more sophisticated, methods than ours. Classic HL tissues are rich in ribonucleases (in eosinophils!). MV-RNAs was of low abundance. GAPDH with its abundant RNA may not be an adequate choice for a housekeeping gene in this experiment. Our 7 cases studied in Germany were all MC-HL, most were EBV positive. We had studied 5 for MV-RNA. They were faint (2) or negative (3). A possible additional evidence of a role for MV in carcinogenesis is that MV proteins are capable of interacting and stabilizing the Pirh2 protein, and ubiquitin E3 ligase by preventing its ubiquitination. Pirh2 negatively regulates $\mathrm{p} 53$ by targeting it for degradation in the proteasome.

\section{"Recent history": Mv association with other cancers: Endometrial carcinoma and measles virus}

We studied 49 cases of EC, 36 with known follow-up. 26/36 (72\%) expressed MV antigens in tumor cells, mainly in type I EC. 16/21 were positive for MV-RNA by ISH. When type II EC was allied with MV-deeper myometrial invasion and a higher mortality were evident [1] (Figure 6). 


\section{Lung cancer and measles virus antigens expression}

a) MV-P protein inhibits the function of Pirh2 [2].

b) 65 NSCLC diagnosed within 10 years.

c) 54/65 were positive for MV antigens associated with older patient age and positive Pirh2 expression - as well as with improved survival [3].

\section{Breast cancer and the measles virus}

a) $64 \%$ of 131 cases were positive for MV antigens by IHC

b) MV antigens expression was associated with ER ( $\mathrm{p}=.018)$; with Ki-67<40\% ( $\mathrm{p}=.029)$; with low or intermediate grade ( $\mathrm{p}=.037)$; with age $<50(p=.039)$; with p53 over-expression ( $p=.049)$.

\section{Breast cancer, conclusions}

a) Evidence of MV antigens expression in a high proportion of breast cancers

b) MV association with younger age, lower histologic grade and p53 overexpression suggests it may play a role in the development of breast cancer [4] (table 4).

\section{Are there MV-negative cancers?}

The non-Hodgkin lymphomas tested with cHL (25) were negative for MV antigens. But ALK1-positive anaplastic large cell lymphomas were positive. Seminomas showed in a few cases background staining for MV antigens, but they were negative.

\section{Additional cancers negative for measles virus}

Prof. Samuel Ariad - communication:
a) Glioblastomas
b) Mesotheliomas

Additional study in isolated tumors:
a) Merkel cell tumors
b) Hepatocellular carcinoma
c) MMMT
d) Desmoid tumor

A Breakthrough? apoptosis of HRS cells in cHL revisited [5].

We reviewed the role of apoptosis in HRS cells of classic HL, in the light of conflicting evidence. We found that HRS cells showed inhibition of apoptosis in 55\% of the 217 cases only.

It is also suggested that NF- $\mathrm{KB}$ (p65) and LMP1/EBV do not correlate with apoptosis inhibition, in contrast with the consensus view.
Apoptosis of HRS cells (2): The most significant association of HRS cell apoptosis was with p53,

the negative expression of which was related with a high apoptotic index $(p=.001)$. We analyzed the relationship between positive MV expression and factors related with apoptosis and found associations with apoptotic index less than median ( $\mathrm{p}=.005)$; with Mdm2+ ( $\mathrm{p}=.028)$; with IкB+ $(\mathrm{p}=.0001)$.

Apoptosis of HRS cells - conclusions: Apoptotic index lower than median was related with

positive MV expression, as was a positive Mdm2 expression. Viruses have a propensity to modulate apoptotic mechanisms in the infected cell. We believe that our demonstration of a pathogenic mechanism involving MV in cHL may favor a causal role for MV, at least in $\mathrm{cHL}$.

WHO report on measles - 2011: In the first 9 months of 2011, 26,000 new cases of measles in

Western Europe. 9 deaths and 7,288 hospitalizations. At the same time-100,000 cases were recorded in the Democratic Republic of Congo. 90\% of European cases are in adolescents and adults, without vaccination, or with an inadequate one. Comeback of measles to Europe is feared.

\section{Provisional Conclusions}

A relation between MV expression and several malignancies is displayed. In cHL this may even be causally related with apoptosis modulation as the supportive mechanism. Findings are probably sustained by an increased cHL morbidity related with a raised measles occurrence in young adults, in Israel [4] in Quebec and in Connecticut. This may be due to frequent outbursts of measles.

Studies in collaboration with Ariad S, Sacks M, Shemer-Avni Y, Myint YY, Levy A, Mejirovski E, Klinkovich I, Gopas J

Many thanks!

\section{References}

1. Pirh2 (2004) The E3 ubiquitin ligase is expressed in lung cancer and inhibits p53. J Natl Cancer Inst 96: 1718-1721.

2. Benharroch et al. (2009) Eur J Obstet Gynecol Reproductive Biol 147: 206-209.

3. Sion-Vardy et al. (2009) Exp Lung Res 35: 701-712.

4. Ariad et al. (2011) Anticancer Res 31: 913-920.

5. Benharroch et al. (2010) APMIS 118: 339-345 